\title{
Urban maintenance for sustainable cities
}

\author{
M. Di Sivo \& D. Ladiana \\ Department of Technologies for Built Environment, \\ University "G. d'Annunzio" of Chieti and Pescara, Italy
}

\begin{abstract}
The attention paid to the themes of curing and maintaining represents one of the most significant acquisitions in post-industrial contemporary culture, which is more and more oriented towards sustainability objectives. The urban maintenance theme can be considered as a real strategy for built environment sustainability; indeed, it is the primary tool for gaining knowledge about and governing the complex and heterogeneous contemporary reality, through which we can achieve system reliability, as well as sustainability regulating the life dimension. This contribution becomes an opportunity for reflecting within the technological culture of a maintenance project whose disciplinary discussion is today based on the application modes of such a discipline at both a building and an urban scale. Indeed, the methods and instruments for programming and maintaining the urban organism - intended as a complex unit system - cannot be those ones used for sub-systems and urban components; they have to be strategic instruments that are able to help managers and citizens to "decide how to decide". It is necessary to reach an integrated approach that, overcoming the logic of a technical management intended as a mere addition of parts, reaches systemic operating modes.

Keywords: sustainable development, urban process, strategic planning, urban maintenance, technological culture.
\end{abstract}

\section{Introduction}

The challenge of development sustainability characterizing contemporary society asks one to re-think, in all sectors, the forms of planning, programming and projecting in cultural, scientific and operational terms, in order to obtain politics, tools, approaches and technologies that are able to allow for anthropic systems 
management and transformation, guaranteeing the present exigencies fulfilment without compromising such a chance in the future.

Such a condition requires a substantial re-thinking of the development philosophy, and in particular of the traditional public investments supporting the economy, while in the industrial society the State used to intervene, investing in infrastructures in order to create an external economy complex and activating conditions to develop private investments - in the new post-industrial context the optic must substantially change. Once any dimensional expansion of infrastructures is defined as impossible - because of the substantially immaterial character of the development processes - the role of the State has to be efficiency-oriented towards the existing structures, by operating those interventions that permit one to maintain and improve conditions. In other words, the type of public intervention has to pass from a diachronic dimension, typical of the industrial model, to a synchronic one. In the first case, the exigency of actuating large infrastructural investments had found its reason in the necessity to create a completely new system of connections through which to start the system take off phase; in the post-industrial reality, the synchronic interval becomes a sustaining action in order to humanize and re-qualify the existent systems. Indeed, while in the industrialization phase both the private and the public investments were implemented without considering the possible effects in terms of territorial and social balance, in the new post-industrial reality it is fundamental to change this kind of approach. After a turbulent and disordered development phase, during which many mistakes were made, new problems have now arisen, not only for future generations but also for our daily life, which is more and more menaced by the consequences of the many senseless politics carried out up to now.

As suggested by the philosopher Hans Jones, we should carry out a completely new action of modifying and managing the environment as a process based on the adoption of a care and responsibility attitude. The theme is particularly critical for those subjects involved in the technology process. The technological culture, from a tool related to transformation activities, has to evolve into a guide in those processes aiming at objectives that overcome the current quantitative and materialistic conception of contemporary society, towards new balances within the global system.

Technology, from being an element of progressive and potentially irreversible unbalance between man and nature, and between man and the consequences of its own production, has to be reconverted into a balancing factor by means of a continuous critical exam of techniques, in order to rule the phenomena following their use.

In such a perspective technology, redefined in disciplinary terms as "the doctrine of transformation processes, generally aiming not only at guiding but also at motivating such processes by correctly answering, in an ecological perspective, to the question: who is it useful to?" (Ciribini [2]), represents a tool made by rules and procedures useful to a human development which is in harmony with nature, achieving in such a manner ethical results. 
Indeed, the right purpose of technology is not to dominate or manipulate nature, but to live in harmony with it.

It is actually important to underline that recovering a correct relationship between man and the built and natural environment can be reached by consciously using the technological tool and its evolution.

Quality, safety and time are critical categories to be used as bases for a development epistemology since today, in such an ecological and systemic perspective, they represent the real values to take into account both in the scientific evolution and in the choices linked to a sustainable development.

The usefulness of research in the field of architecture should be mostly carried out by assuming the fundamental role of guide of the processes of anthropic change.

\section{Care and maintenance}

It is evident the need to take care of natural and built environment as well: indeed, it is necessary to exploit only those resources really needed, to produce less waste and pollution, to care and maintain slopes, watersheds, coasts, as well as public green spaces, streets, infrastructures, etc (the list could be very long).

Caring about something means "being concerned about it, looking after it, but at the same time being ready to act, put our ideas into action" (Preta [13]).

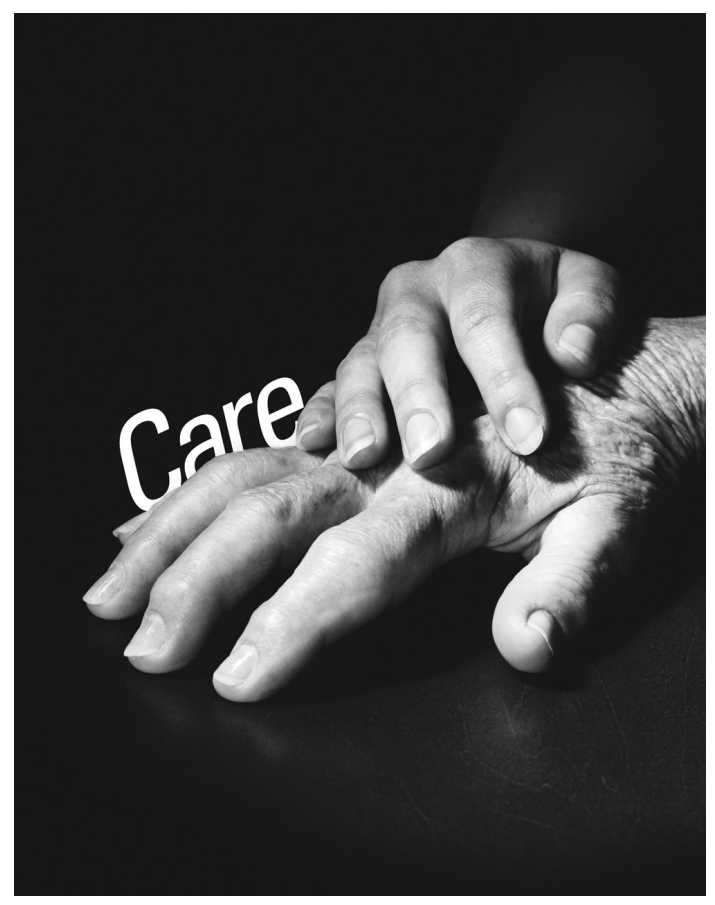

Figure 1: Caring about something means being concerned about it. 
Care consists of actions performed in a responsible way, elicited by a careful attention upon the object.

Contemporary culture, based on the post-positivist heritage, was forced to conceive "care" only in relation to its results (according to a habitual reason: cause-effect); anyway, our culture should be brought back to its original process meaning: care as the only way to be able to direct and follow the object transformation processes over time, being not only a tool to recompose a structure subjected to irreversible transformations but also to recompose evolutionary transformations, the same as "mother cares".

The issue of object care has been described in the famous work by Pirsig [12], where it is underlined how it is not sufficient a purely "rational" approach in any maintenance problem: "(...) it does not exist any guide about the essential issue related to a motorbike maintenance: caring about it. This is considered scarcely important, or it taken for granted".

Only care, assuming a different and higher relationship between man and the product universe he is the prime mover, will be able to guarantee that character of permanence overt time: project care, construction care, maintenance care; care incorporates time and transmits it to objects.

By searching for the character of "permanence" and "resource conservation", it is possible to re-discover the important relationship with our assets and urban environment, by means of a renewed approach to maintenance, intended as a discipline critical to the regulation of complex systems, ad aiming not only to the efficiency of such systems but also to the conservation of the ecosystem balance.

\section{Maintenance for a sustainable city}

Translating the notion of development sustainability into an urban dimension is not a simple enterprise, both for the complexity of the studied object and for the theoretical and practical implications such translation causes in constructing and managing a sustainable city.

In order to develop strategies and policies of city sustainability, it is necessary to start from the evaluation of its specificities, firstly from its total artificiality: maybe the city represents the most important product of the technological and cultural evolution of man - aimed to maximise social interaction - within which resources are few, controlled and used for specific purposes; such a consideration highlights the contradiction of those attempts of mechanical transposition of the concept of city developed in order to environmental sustainability and based on the economy of natural resources.

So, if the city represents an artificial environment - whose nature expresses itself through the co-existence and integration of social, economic and physicalenvironmental systems (natural or built) - the concept of sustainability should be defined by such an assumption; by pursuing specific objectives having a localurban character (decrease in pollution, noise, degradation and obsolescence of built environment), once such specific objectives are faced and fixed they could contribute to achieve global objectives. 


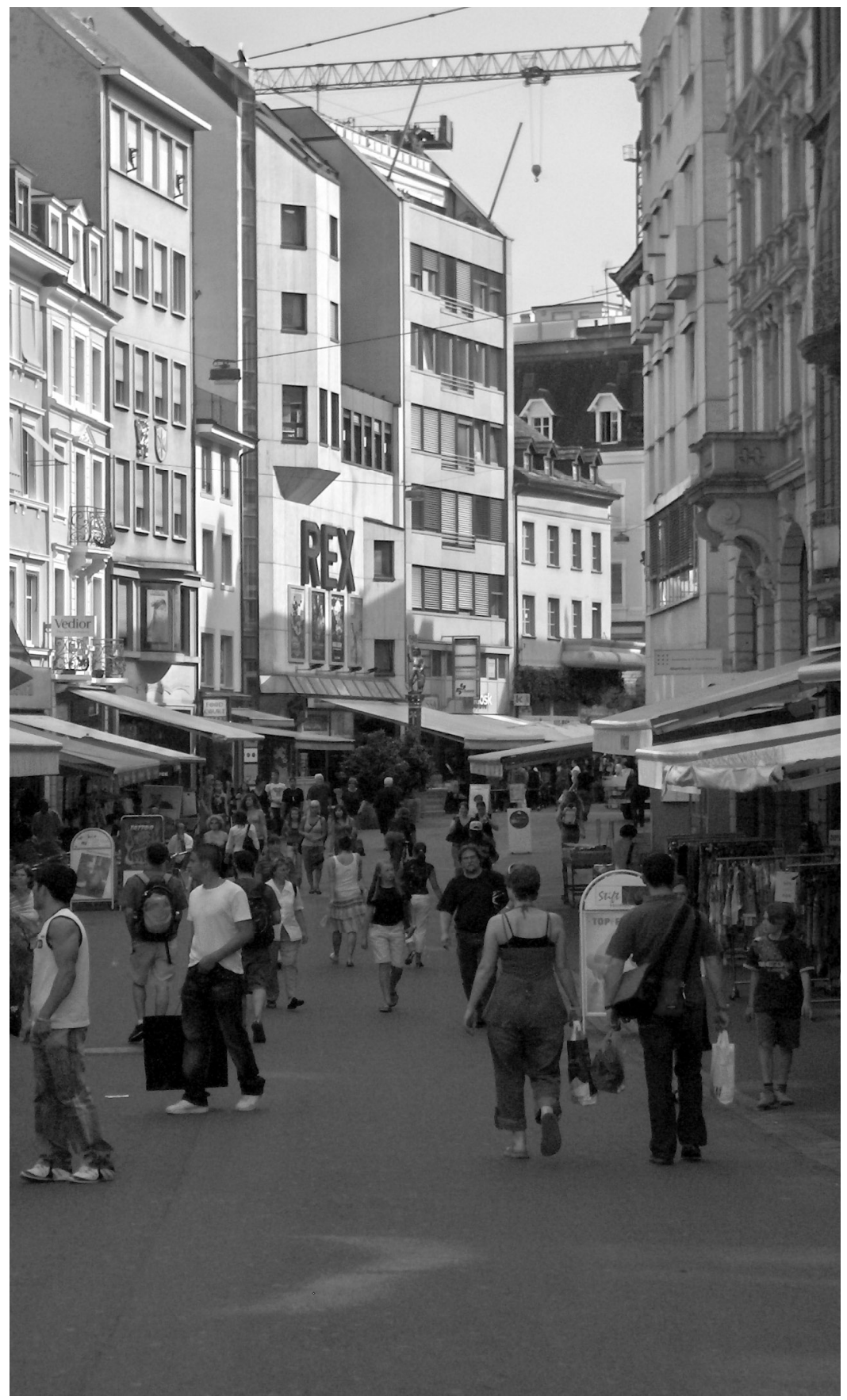

Figure 2: $\quad$ The city as a resource. 
The quest to attract urban sustainability involves accepting city as a "resource", since such a capital, having an anthropic origin and so rich in value and history, has been accumulated thanks to the exploitation of natural capitals. Such an approach can contribute to achieve (even social) development aspirations, provided that the capital and the fluxes of natural resources are not exploited and degraded so as to compromise their future usefulness.

The city, "unsustainable" by definition, in such a view aims to sustainability objectives, being intended as a resource, a place where to express the material culture of a civilization, place where opportunities, values, information, events and social, economical and cultural life are joined together.

The challenge of city care involves assuming urban development sustainability as the scenario within which the adequate levels of care, maintenance-conservation and consumption have to compare themselves with development aspirations, but at the same time the consumption/substitution of capitals made of urban resources should be coherent with the exigency of maintaining natural resources as well as those resources generated by anthropic action; such a challenge will have to be won by identifying new forms of urban policy, recognizing and enhancing the value of the role of maintenance for preserving the physical-natural-cultural heritage, by means of scientific processes of planning and control and by carrying out adequate action strategies.

\section{Methods and tools for asset and maintenance management}

The challenge characterizing the governing of contemporary city consists then searching, elaborating and experimenting specific tools to govern interventions in assets.

The problem of optimizing the urban asset management modes through a careful planning presents urgency because of several causes: the progressive decrease of the Public Administration financial availability concurrently at important increases at level of properties, the major attention users pay to the quantity/quality of erogated services, their interest in public health and safety involving a renewed attention to themes such as air and water quality, green spaces, traffic and noise decrease, progressive ageing of people resulting in a more difficult city practicability and access to services, the obsolescence of many infrastructures built during the urban growth, the increase in the social and economic costs to manage and maintain environmental resources; these are signals that call for reflecting upon the implementation modes of urban system and services.

The necessity to leave policies based on emergency management and fragmentary planning actions based on distinct sectors becomes more evident and urgent with those urban systems characterized by a political and social desire to perform planning and management policies aiming to pursue a sustainable city, as well as by obsolescent or degraded infrastructures setting up the city consolidated parts.

The theoretical debate on the development of shared tools to manage and control urban maintenance still has difficulty getting out of a technical-operative 


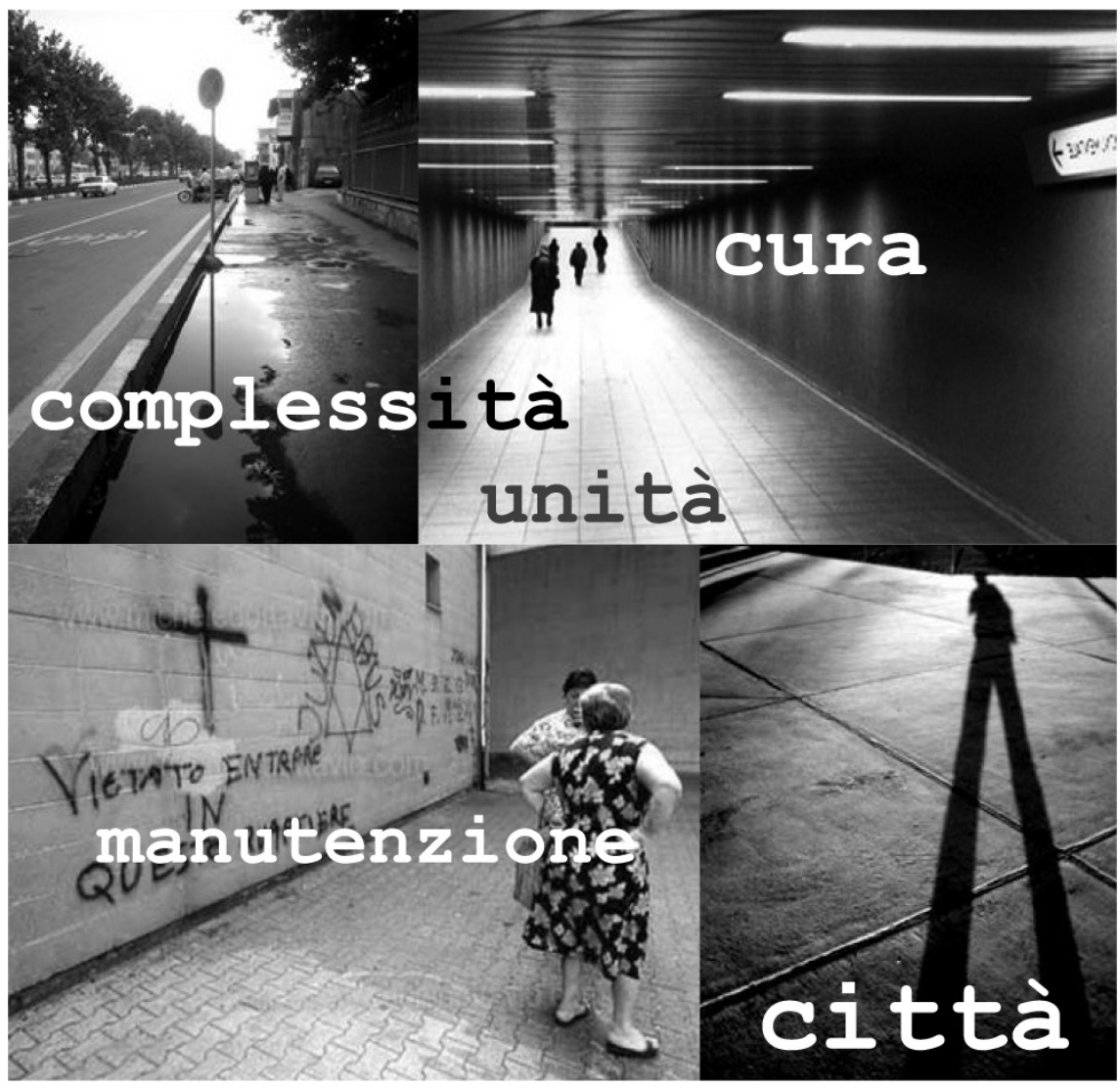

Figure 3: $\quad$ Obsolescent or degraded infrastructures.

context - deriving from industrial culture - in order to usefully compare itself to methodologies belonging to different disciplinary contexts. Indeed, the methods and tools of planning and managing the urban environment - conceived as a complex unitary system - can be assumed as substantially different from those definable as technical-operational methods for planning and managing parts or sub-systems (widely used in the maintenance culture more than in the maintenance praxis); in other words, it consists in "how and when to do something": performing maintenance on an urban scale consists in the preparation of strategic tools capable of helping managers and citizens to "decide how to decide".

It is then necessary to develop methodologies and tools aimed to assist Public Administrations to identify needs, evaluate solutions and plan long term sustainable strategies, in order to reach a better performance of the city system minimizing at the same time economic and environmental costs.

It is apparent that we are talking about methods and tools for the optimization of decisional processes, in order to devote financial, human and environmental 
resources to a continuous improvement of sustainability of urban systems as well as of their performances and levels of service.

An adequate planning enhances the achievement of important - based on the new need - the systems characterized by obsolescence.

Such a planning could also guide the action of management policies of urban systems by means of programmes aiming to regulate the variable of infrastructure and services demand, trying to change the user behaviour: promoting the use of public transit as well as of private transit having a lower environmental impact, encouraging programmes for reducing the water resources exploitation, etc.

It emerges then the need to plan management actions of building systems which take adequately account not only of the technical instances but also of the social, cultural and economical ones.

A possible answer can come from integrating the maintenance process to the strategic planning process, in order to pursue objectives over an adequate time horizon, based on strong tools for evaluating and classifying priorities, as well as on policy involving all subjects - including citizens/users - and all levels characterized by decisional processes within Public Administration.

Public participation to the working out of strategies, not only represents an answer to the increasing need of transparency in city management, but it also involves a deep understanding of the citizens' needs and expectancies, as well as the necessary consent to the planning sources.

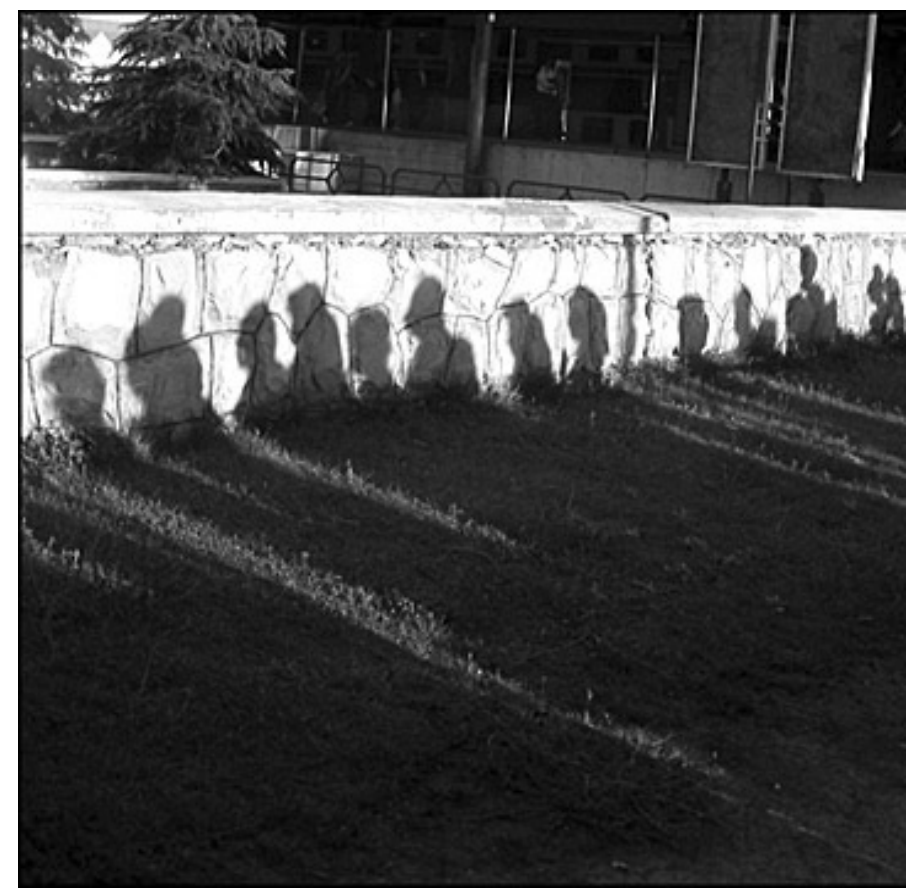

Figure 4: Public participation is important for the working out of strategies. 
The strategic planning is a complex process, consisting in: rational analysis of mandates and missions, creation of an object system, selection of the benchmarks associated to each objective, analysis of individual activities and their related human, technical and economic tools (the analysis is also defined "re-engineering" process), reconstruction of expense budget (by program, by objective and by performance), and, finally, creation of a permanent system controlling both the projected rhythms and goals and the defined and adopted standards.

The importance of implementing the urban system maintenance programming within the strategic planning process can be understood not only thanks to the substantial improvement of the maintenance decisional process - resulting from being integrated into the more complex system of city programming and managing - but also for its highlighting how, within the city objective system, the efficiency system of urban structure (streets, infrastructures, green, etc) becomes a cross objective strongly affecting the ways of pursuing different objectives; indeed, strategic objectives such as sustainability, safety or built environment quality, can't be achieved without implementing maintenance operating objectives.

It is such a chance of optimizing the achievement of integrated objectives that, for city environmental sustainability purposes, makes the implementation of urban maintenance under the strategic planning important; indeed, an important challenge deriving from this connection will have to be the development of an integrated approach that, overcoming the logics of a technical management intended as a mere part summation, reaches systemic operational modes capable of minimizing the impact on environment.

For example, within such a vision the achievement of integration, in programming new constructions and maintaining obsolete or degraded parts of street networks or sub-services, there may be important advantages such as keeping a high level of service or minimizing life cycle costs, environmental impact and traffic disruption or residents annoyances.

Losses and breaks on water mains can result in breaks or degradation on the nearby wastewater mains or street network; at the same time, the sewage deterioration can undermine the nearby water and street structure; the vibrations generated during street rehabilitation/reconstruction can cause the underground structures collapse; the fluxes of electric energy from electric lines, railways or cathodic protection systems can accelerate the electrolytic corrosion of metal pipes.

Pursuing integration in construction and maintenance of linear infrastructure is a difficult but indispensable task to be able to overcome the irrationality of current operating modes, characterized by a substantial disinterest in the effects of mutual interference among systems and by a great waste of economic and environmental resources; such an important objective can represent a specific contribution on an urban scale from maintenance programming and management, aiming to raise performance and lifetime levels of network systems, and to achieve a sustainable city. 


\section{Acknowledgements}

Michele Di Sivo is the author of sections 1 and 2; Daniela Ladiana of sections 3 and 4 .

\section{References}

[1] Ashby, W. R., Introduzione alla cibernetica, Einaudi, Torino, 1971.

[2] Ciribini, G., Introduzione alla tecnologia del design, Franco Angeli, Milano. 1979.

[3] Di Sivo, M. \& Ladiana, D., Sicurezza e manutenzione dell'ambiente costruito, Alinea Editrice, Firenze, 2007.

[4] Di Sivo, M., Il progetto di manutenzione, Alinea, Firenze, 1992.

[5] Di Sivo, M., Manutenzione Urbana, Alinea, Firenze, 2004.

[6] Dioguardi, G., I sistemi organizzativi, Bruno Mondadori, Milano. 2004.

[7] Dioguardi, G., Manutenzione d'eccellenza come strategia d'innovazione, In XX Congresso Nazionale AIMAN (Bologna, 20-21 February), 2003.

[8] Ferracuti, G., Tempo, qualità, manutenzione. Scritti sulla manutenzione edilizia, urbana e ambientale, Alinea, Firenze, 1994.

[9] Ladiana, D. (a cura di), Manutenzione e gestione sostenibile dell'ambiente urbano, Alinea Editrice, Firenze, 2007.

[10] Ladiana, D., Pianificazione della gestione e manutenzione delle infrastrutture urbane. L'approccio top-down e bottom-up, Alinea Editrice, Firenze, 2008.

[11] Jonas, H., Il principio responsabilità. Un'etica per la civiltà tecnologica, Einaudi, Paperback, Torino, 1990.

[12] Pirsig, R M., Lo Zen e l'arte della manutenzione della motocicletta, Adelphi, Milano, 1981.

[13] Preta, L., In principio era la cura, Editori Laterza, Bari, 1995. 